University of Nebraska - Lincoln

DigitalCommons@University of Nebraska - Lincoln

Characterization of Triacylglycerols From Overwintering Prepupae of the Alfalfa Pollinator Megachile rotundata (Hymenoptera: Megachilidae)

\author{
James S. Buckner \\ USDA-ARS, bucknerj@fargo.ars.usda.gov \\ William P. Kemp \\ USDA-ARS \\ Jordi Bosch \\ Utah State University
}

Follow this and additional works at: https://digitalcommons.unl.edu/usdaarsfacpub

Buckner, James S.; Kemp, William P.; and Bosch, Jordi, "Characterization of Triacylglycerols From Overwintering Prepupae of the Alfalfa Pollinator Megachile rotundata (Hymenoptera: Megachilidae)" (2004). Publications from USDA-ARS / UNL Faculty. 1431.

https://digitalcommons.unl.edu/usdaarsfacpub/1431

This Article is brought to you for free and open access by the U.S. Department of Agriculture: Agricultural Research Service, Lincoln, Nebraska at DigitalCommons@University of Nebraska - Lincoln. It has been accepted for inclusion in Publications from USDA-ARS / UNL Faculty by an authorized administrator of DigitalCommons@University of Nebraska - Lincoln. 


\title{
Characterization of Triacylglycerols From Overwintering Prepupae of the Alfalfa Pollinator Megachile rotundata (Hymenoptera: Megachilidae)
}

\author{
James S. Buckner, ${ }^{1 *}$ William P. Kemp, ${ }^{2}$ and Jordi Bosch ${ }^{3}$ \\ Alfalfa leafcutting bees, Megachile rotundata (F.), overwinter as prepupae. The internal lipids were extracted from prepupae \\ that had been wintered at $4^{\circ} \mathrm{C}$ for 7 months. Megachile rotundata prepupae possessed copious quantities of internal lipids \\ (20\% of the fresh weight) that were extracted with $\mathrm{CHCl}_{3}$ /methanol (2:1). Transmission electron microscopy revealed that \\ lipids were stored within very large intracellular vacuoles. Separation by silica chromatography revealed that $88 \%$ of the \\ internal lipids were triacylglycerols. Ester derivatives of fatty acids from triacylglycerol components were analyzed by gas \\ chromatography-mass spectrometry and 15 fatty acid constituents were identified. The majority $(76 \%)$ of the triacylglycerol \\ fatty acids were unsaturated fatty acids. The major triacylglycerol fatty acid constituent (30\%) was the $C_{16}$ monounsaturated \\ fatty acid, palmitoleic acid (16:1, hexadec-9-enoic acid), with substantial amounts of linolenic acid (18:3, octadec-9,12,15- \\ trienoic acid, 15\%), palmitic acid (16:0, hexadecanoic acid, 14\%) and oleic acid (18:1, octadec-9-enoic acid, 13\%). Palmitoleic \\ acid as the major fatty acid of an insect is an unusual occurrence as well as the presence of the 16-carbon polyunsaturated \\ fatty acids, 16:2 and 16:3. The major intact triacylglycerol components were separated and identified by high performance \\ liquid chromatography-mass spectrometry. A complex mixture of approximately 40 triacylglycerol components were identified \\ and major components included palmitoyl palmitoleoyl oleoyl glycerol, palmitoyl palmitoleoyl palmitoleoyl glycerol, myristoyl \\ palmitoleoyl palmitoleoyl glycerol, myristoleoyl palmitoyl palmitoleoyl glycerol, and palmitoyl palmitoleoyl linolenoyl glycerol. \\ The function of these internal lipids and their relevance to winter survival and post-wintering development of $M$. rotundata is \\ discussed. Arch. Insect Biochem. Physiol. 57:1-14, $2004 . \quad$ Published 2004 Wiley-Liss, Inc. ${ }^{\dagger}$
}

Keywords: alfalfa leafcutting bee; storage lipids; unsaturated fatty acids; palmitoleic acid

\section{INTRODUCTION}

The alfalfa leafcutting bee, Megachile rotundata (F.) is a summer-flying, cavity-nesting, solitary bee. This species is the pollinator of choice for alfalfa,
Medicago sativa L., seed production throughout western North America (Peterson et al., 1992) and much emphasis has been placed on the development of wintering procedures and improved incubation schedules to synchronize the emergence of

\footnotetext{
'Biosciences Research Laboratory, USDA-ARS, Fargo, North Dakota

${ }^{2}$ Bee Biology \& Systematics Laboratory, USDA-ARS, Logan, Utah

${ }^{3}$ Department of Biology, Utah State University, Logan, Utah
}

Mention of trade names or commercial products in this article is solely for the purpose of providing specific information and does not imply recommendation or endorsement by the U.S. Department of Agriculture. U.S. Department of Agriculture, Agricultural Research Service, Northern Plains Area, is an equal opportunity/ affirmative action employer and all agency services are available without discrimination.

Abbreviations used: BSA = N,0-bis-(trimethylsilyl) acetamide; FAME = fatty acid methyl ester; $L=$ linoleic acid; $L a=$ lauric acid; $L n=$ linolenic acid; $M=$ myristic acid; $M_{0}=$ myristoleic acid; $0=$ oleic acid; $P=$ palmitic acid; $P_{0}=$ palmitoleic acid; $P_{L}=$ palmitlinoleic acid; $P_{L n}=$ palmitlinolenic acid; $S=$ stearic acid; $T A G=$ triacylglycerol; $t$-BME $=$ tert-butylmethylether; $T M S=$ trimethylsilyl.

*Correspondence to: James S. Buckner, USDA-ARS, Biosciences Research Laboratory, 1605 Albrecht Blvd., Fargo, ND 58105.

E-mail: buckneri@fargo.ars.usda.gov

Received 1 August 2003; Accepted 2 May 2004

Published 2004 Wiley-Liss, Inc. TThis article is a US Government work and, as such, is in the public domain in the United States of America. DOI: 10.1002/arch.20008

Published online in Wiley InterScience (www.interscience.wiley.com) 
M. rotundata with alfalfa bloom (Johansen and Eves, 1973; Taséi and Masure, 1978; Richards et al., 1987; Richards and Whitfield, 1988; Murrell, 1991; Kemp and Bosch, 2000). At most North American latitudes, $M$. rotundata emerge and mate during June and July, and female bees build nests in abandoned beetle burrows in timber, in cavities associated with farm buildings, or artificial nesting materials provided by alfalfa seed producers (Stephen, 1981; Rank and Goerzen, 1982; Richards, 1984). Nests consist of a linear series of cells constructed from cut-leaf pieces. Each cell is provisioned with a mass of pollen and nectar, on top of which an egg is deposited. Completed nests are sealed with plugs of leaf cuttings. By mid-summer, fifth-instar M. rotundata completely consume their provision, defecate, and spin a cocoon. In this stage (prepupa), most individuals undergo a dormant period that lasts through the winter. Alternatively, some individuals may pupate within the summer and emerge as a partial second generation, whose progeny winter as prepupae (Parker and Tepedino, 1982; Rank and Rank, 1989; Kemp and Bosch, 2001).

A recent study has shown that respiration rates of $M$. rotundata significantly decline within 7 days after cocoon completion and remain low throughout late summer and winter (Kemp et al., 2004). Other evidence that prepupal dormancy in $M$. rotundata is diapause-mediated comes from empirical results showing that prepupae artificially wintered for insufficiently long periods take much longer to resume development after being exposed to incubation temperatures (Johansen and Eves, 1973; Taséi and Masure, 1978; Richards et al., 1987). Other studies have addressed glycerol production and supercooling points in M. rotundata prepupae (Gusta, 1982; Krunic et al., 1982). Beyond these studies, we know little about the overwintering physiology of $M$. rotundata. A better understanding of the physiological processes underlying the summer-winter dormancy of this species is important because most Megachilidae (Bosch et al., 2001), and most Hymenoptera (Danks, 1987; Gauld and Bolton, 1988) overwinter as prepupae. Ultimately, this information is also important to understand winter mortality in $M$. rotundata and helps improve management protocols to optimize the development, health, and synchronized emergence of this important alfalfa pollinator (Bosch and Kemp, 2002).

For insects overwintering in temperate regions (frequently in a non-feeding diapause condition), energy required for winter survival, post-diapause development, and the emergence of adults comes from a variety of intermediary metabolites, including stored internal lipids (Downer, 1985; Lee and Denlinger, 1991). Accumulation of triacylglycerols (TAGs) as energy reservoirs and an increase in the ratio of unsaturated/saturated fatty acids has been reported for several overwintering temperate insects (Kurthiala and Chippendale, 1989; Ohtsu et al., 1993, 1995; Joanisse and Storey, 1996). In this study, the first in a series of investigations elucidating relevant aspects of $M$. rotundata developmental physiology, internal lipids were extracted from diapausing $M$. rotundata prepupae that had been wintered at $4^{\circ} \mathrm{C}$ for 7 months. Internal lipids were quantified and characterized by thin-layer chromatography (TLC), silica column chromatography, gas chromatography-mass spectrometry (GC-MS), and high performance liquid chromatography-MS (HPLC-MS).

\section{MATERIALS AND METHODS}

\section{Materials}

Silica (Porasil $125 \AA$ ) was purchased from Waters Corp., Franklin, MA. High-performance thinlayer plates $(10 \times 10 \mathrm{~cm}, \mathrm{E}$. Merck, silica gel 60$)$ were purchased from EM Science, Gibbstown, NJ. Authentic lipid standards were purchased from NuChek Prep, Inc., Elysian, MN. Tertiary-butylmethylether and pyrrolidine were purchased from Aldrich Chemical Co., Inc., Milwaukee, WI. The 10\% $\mathrm{HCl}$ in methanol reagent was prepared in-house by bubbling gaseous $\mathrm{HCl}$ into redistilled methanol. Reactivials ${ }^{\circledR}, \mathrm{N}, \mathrm{O}$-bis-(trimethylsilyl) acetamide (BSA), and dimethylformamide were purchased from Pierce Chemical Co., Rockford, IL. Tousimis/Millonig's buffer $(0.1 \mathrm{M}, \mathrm{pH} 7.35), 2.5 \%$ glutaraldehyde in Millonig's buffer, and Spurr's epoxy resin were pur- 
chased from Tousimis Research Corp., Rockville, MD. Other chemicals were of analytical grade.

\section{Insects and Lipid Extraction}

The diapausing $M$. rotundata prepupae used in this study were from a population maintained at the USDA-ARS Bee Biology \& Systematics Laboratory, Logan, UT, at $4^{\circ} \mathrm{C}$ for approximately 210 days, starting in September 2001. Overwintering, cocooned prepupae, within the nesting cells, were shipped in March 2002 at $4-10^{\circ} \mathrm{C}$ to the USDAARS Biosciences Research Laboratory, Fargo, ND, and held at $4^{\circ} \mathrm{C}$ until use. Prepupae were carefully removed from their cells and their fresh weight was determined. For determination of water content, prepupae were frozen at $-80^{\circ} \mathrm{C}$ and lyophilized for $24 \mathrm{~h}$. The freeze-dried prepupae were then reweighed and the free water content was determined.

Prior to extraction of internal lipids, the cuticular lipids were removed from pooled groups of $M$. rotundata prepupae by submersion in hexane for $60 \mathrm{sec}$, followed by a 30-sec rinse with chloroform $\left(\mathrm{CHCl}_{3}\right)$. The internal lipids were removed from either 5 or $10 \mathrm{M}$. rotundata prepupae by tissue homogenation in $\mathrm{CHCl}_{3} / \mathrm{MeOH}(2: 1)$ and extraction into $\mathrm{CHCl}_{3}$ (Folch et al., 1957). Pre-weighed prepupae were placed in a glass Ten-Broeck tissue grinder. The ratio of volume of extracting solvent to the number of prepupae was $1 \mathrm{ml} \mathrm{CHCl}_{3} / \mathrm{MeOH}$ per prepupa. After sonication in the water bath of an ultrasonic cleaner (Fisher Scientific, Model FS 60) for $45 \mathrm{~min}$, the prepupae were homogenized with the hand-held tissue grinder. The homogenate was transferred into a glass separatory funnel and the lipids recovered in the $\mathrm{CHCl}_{3}$ layer from partitioning the homogenate with water. The water layer was partitioned with additional $\mathrm{CHCl}_{3}$. The combined $\mathrm{CHCl}_{3}$ layers were backwashed once with water and, after evaporating the $\mathrm{CHCl}_{3}$, the gravimetric weight of the lipid residue was obtained before storage under argon at $-20^{\circ} \mathrm{C}$.

\section{Lipid Class Analysis}

For TLC analysis of lipid extracts and lipid fractions, portions were dissolved in $\mathrm{CHCl}_{3}$ and $5 \mu \mathrm{l}$ (usually 0.005- 0.01 insect equivalent) spotted alongside wax ester, fatty aldehyde, TAG, and fatty alcohol standards onto a $10 \times 10 \mathrm{~cm}(150 \mu \mathrm{m})$ silica gel plate. TLC plates were developed in hexane/ethyl ether/formic acid (80:20:1). For visualization of lipid classes, plates were charred by spraying with $5 \% \mathrm{H}_{2} \mathrm{SO}_{4}$ in $95 \%$ ETOH and heating to $200^{\circ} \mathrm{C}$.

The internal lipids of $M$. rotundata prepupae were separated into neutral lipids and free fatty acids using a modification of a silica Sep-Pak column procedure (Hamilton and Comai, 1988). For separation of internal lipids from a pooled sample of 5-10 prepupae, $0.183 \mathrm{~g}$ of silica was packed into a $53 / 4$ " glass Pasteur pipette $(0.5 \mathrm{~cm}$ i.d.) fitted with a small plug of glass wool. The silica was washed and equilibrated by washing with $4 \mathrm{ml}$ of hexane/ tert-butylmethylether ( $t$-BME) (96:4) followed by $12 \mathrm{ml}$ of hexane. The internal lipids were dissolved in hexane $(10 \mathrm{mg} / \mathrm{ml})$, with heating if necessary,

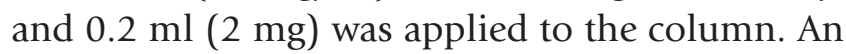
additional $8 \mathrm{ml}$ of hexane, followed by $8 \mathrm{ml}$ of hexane/t-BME (99.5:0.5) was added to remove any surface lipid contaminants (hydrocarbon, wax esters, aldehydes). Triacylglycerols, free fatty acids, and any fatty alcohols were then eluted from the column with successive $8-\mathrm{ml}$ additions of hexane/ $t$-BME (96:4), hexane/acetic acid (100:2), and $t$-BME/ acetic acid (100:0.2), respectively. Column fractions were analyzed for lipid class content and purity by TLC as described above.

\section{Hydrolysis and Formation of Fatty Acid Derivatives}

The fatty acids of TAG fractions were obtained by alkaline hydrolysis with $5 \% \mathrm{KOH}$ in $\mathrm{MeOH}$. Reaction with $10 \% \mathrm{HCl}$ in $\mathrm{MeOH}$ was used to convert fatty acids to their methyl ester derivatives. The methods for hydrolysis and esterification to yield fatty acid methyl esters (FAME) have been previously described (Buckner and Hagen, 2003). Fatty acids were converted to their trimethysilyl derivatives by reaction with BSA. Fatty acids from the alkaline hydrolysis of approximately $50 \mu \mathrm{g}$ of TAG were dissolved in $20 \mu \mathrm{l}$ dry benzene, $20 \mu \mathrm{l}$ dry dimethylformamide, and $60 \mu \mathrm{l}$ BSA. The reaction 
mixture was heated at $70^{\circ} \mathrm{C}$ for $30 \mathrm{~min}$. After cooling, the sample was diluted 10-fold using BSA/DMF/ benzene (20:20:60) and portions analyzed by CGCMS. For determination of double bond location for the fatty acids, the FAMEs from the TAG fraction were converted to their pyrrolidine derivatives. Methods for reaction of FAME with pyrrolidine and recovery of the fatty acid pyrrolidides were as previously described (Buckner and Hagen, 2003).

\section{Gas Chromatography-Mass Spectrometry}

The trimethylsilyl ester derivatives of fatty acids from the TAG fraction were analyzed by GCMS using a 40-meter J\&W DB-1 capillary column (0.18 mm i.d., $0.4 \mu \mathrm{m}$ phase thickness). Operating conditions for the GC-MS analysis for lipid identification and methods for using authentic standards for determination of lipid quantities have been previously described (Buckner et al., 1999; Buckner and Hagen, 2003). Fatty acid pyrrolidides were analyzed using a Hewlett-Packard 5970 GCMS equipped with a Supelco SP-2380 capillary column $(30 \mathrm{~m} \times 0.25 \mathrm{~mm} \times 0.2 \mu \mathrm{m}$ film thickness $)$ and splitless injector at $260^{\circ} \mathrm{C}$. The oven temperature was held at $50^{\circ} \mathrm{C}$ for $2 \mathrm{~min}$, then ramped at $25^{\circ} \mathrm{C}$ per minute to $260^{\circ} \mathrm{C}$ and held $10 \mathrm{~min}$. Helium flow rate was $1 \mathrm{ml} / \mathrm{min}$. Mass spectra data were compared to the mass spectra of the fatty acid pyrrolidides of authentic standards as previously described (Buckner and Hagen, 2003).

\section{High Performance Liquid Chromatography-Mass Spectrometry}

Intact TAGs were separated and identified by HPLC-MS using a Waters Model 2695 Separations Module (Waters Corp., Milford, MA) equipped with a $250 \times 4.6 \mathrm{~mm}$ Chromspher 5 Lipid column (Chrompack, The Netherlands). The column was equilibrated at $1 \mathrm{ml} / \mathrm{min}$ and $30^{\circ} \mathrm{C}$ with hexane/toluene/ethyl acetate (90:5:5), then a linear gradient: to $15 \%$ acetone in $15 \mathrm{~min}$; to $100 \%$ acetone in $10 \mathrm{~min}$; and to $10 \%$ acetonitrile in $5 \mathrm{~min}$. For increased resolution of closely eluting peaks and single peaks containing multiple TAG compo- nents, portions of TAG samples were passed through two Chromspher 5 Lipid columns connected in series. All solvents were dried over molecular sieves before use. Mass spectra of TAG components were obtained using a Waters Integrity System Thermabeam Mass Detector.

\section{Electron Microscopy}

Prepupae were removed from nesting cells and tissues were prepared for transmission electron microscopy by dissection and fixation in ice-chilled $2.5 \%$ glutaraldehyde in Tousimis/Millonig's buffer. The samples were post-fixed in $2 \%$ osmium tetroxide in the same buffer. Following fixation, samples were dehydrated, embedded in Spurr's epoxy resin, and prepared for examination using a JEOL JEM 100CX transmission electron microscope as previously described (Buckner et al., 2002).

\section{RESULTS}

\section{Quantities and Localization of Internal Lipids}

The mean weight per $M$. rotundata prepupa was $48.1 \pm 8.0 \mathrm{mg}$ (mixed sex; $\mathrm{n}=20$ ) and the quantity of extractable internal lipid from a pooled group of $20 \mathrm{M}$. rotundata prepupae was $9.9 \mathrm{mg}$ per prepupa (lipid as \% of fresh weight $=20.6 \%$ / prepupae). TLC analysis of a portion of the internal lipid extract showed only one major band that co-migrated with authentic TAG standard (data not shown) and a minor band at the origin (likely phospholipid). Portions of the internal lipids were fractionated by silica column chromatography, and $88 \%$ of the lipid applied to the column was recovered in the TAG fraction following elution with hexane/t-BME (96:4). The remainder of the internal lipid fraction was likely polar lipids (phospholipids) that were not eluted by silica column chromatography.

Megachile rotundata prepupae were fixed in a buffered glutaraldehyde solution, and internal tissues were excised and prepared for examination by transmission electron microscopy. Staining of ultra-thin tissue sections revealed the presence of 
lipid storage vacuoles within presumably fat body cells (Fig. 1). Within each cell, storage vacuoles were numerous and many were very large (20-25 $\mu \mathrm{m}$ in diameter).

\section{Characterization of Fatty Acids From Triacylglycerols}

A typical GC-MS chromatogram of TMS derivatives of fatty acids from the TAG fraction is shown in Figure 2 and 15 fatty acids were identified. Percentage composition values were determined from integrated peak areas and comparisons to standard curves for TMS derivatives of authentic fatty acids. The majority of the TAG fatty acids were unsaturated fatty acids $(76 \%)$ and the major constituent $(29.5 \%)$ was the monounsaturated fatty acid, palmitoleic acid (16:1), with lesser amounts of other monounsaturated acids, i.e., oleic acid (12.9\%) and myristoleic acid (8.2\%) (Table 1). Also present were the $\mathrm{C}_{18}$ polyunsaturated fatty acids, linoleic acid (5.0\%) and linolenic acid (14.8\%), and small amounts of the $\mathrm{C}_{16}$ counterparts, 16:2 and 16:3. TMS derivatives of fatty acids from the TAG fraction also included the saturated fatty acids, lauric (1.2\%), myristic (7.8\%), palmitic (13.9\%), and stearic $(0.6 \%)$.

The position of double bonds in the unsaturated fatty acids from TAG was determined by GCMS analysis of their pyrrolidine derivatives as previously described (Buckner and Hagen, 2003). For the mass spectrum of an unsaturated component, if an interval of 12 atomic mass units is observed between the most intense peaks of clusters of fragments containing $n$ and $n-1$ carbon atoms

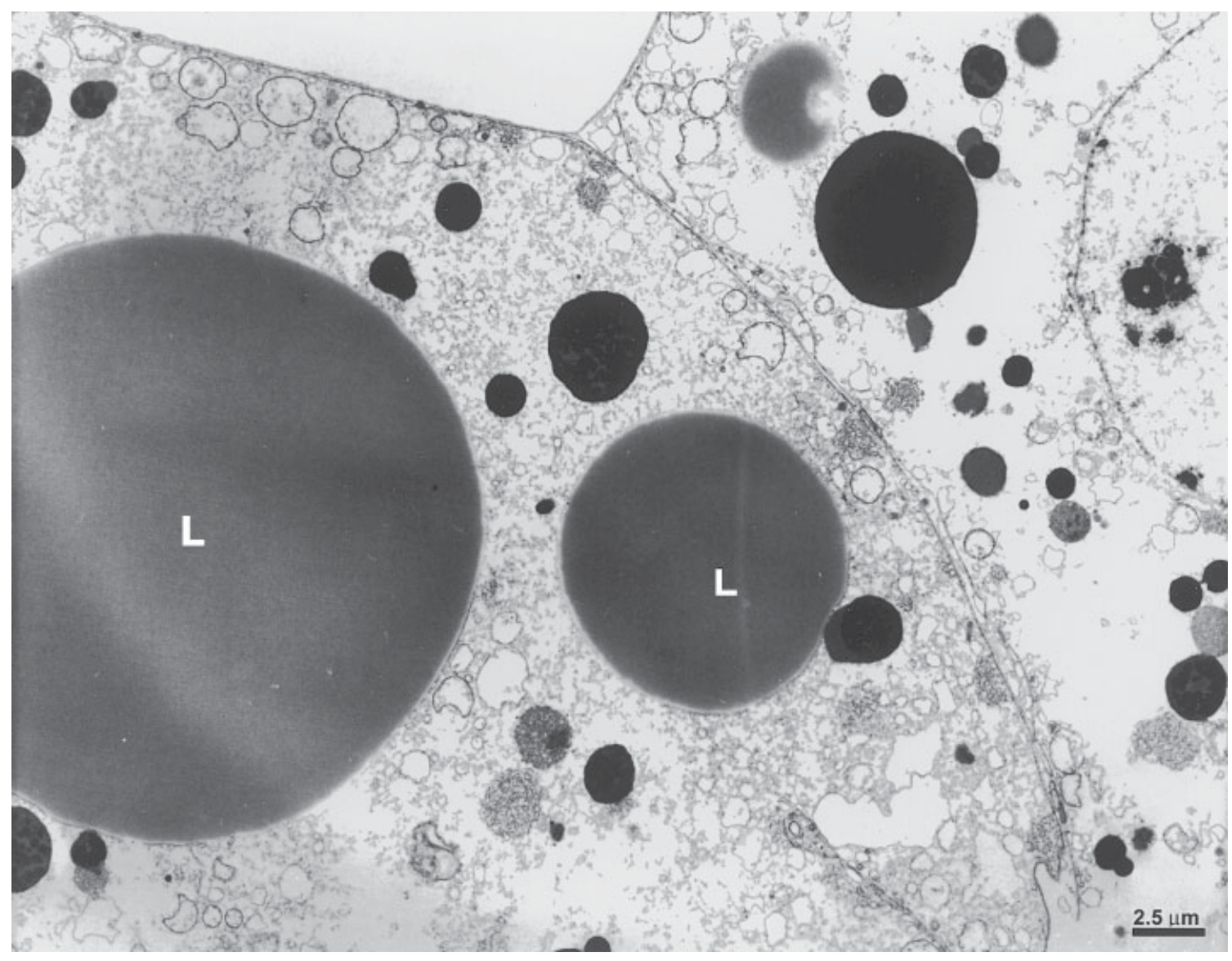

Fig. 1. Transmission electron micrograph of fat body cells from $M$. rotundata prepupae. Cells contained numerous and very large (20-25 $\mu \mathrm{m}$ diameter) lipid storage vacuoles (L). 


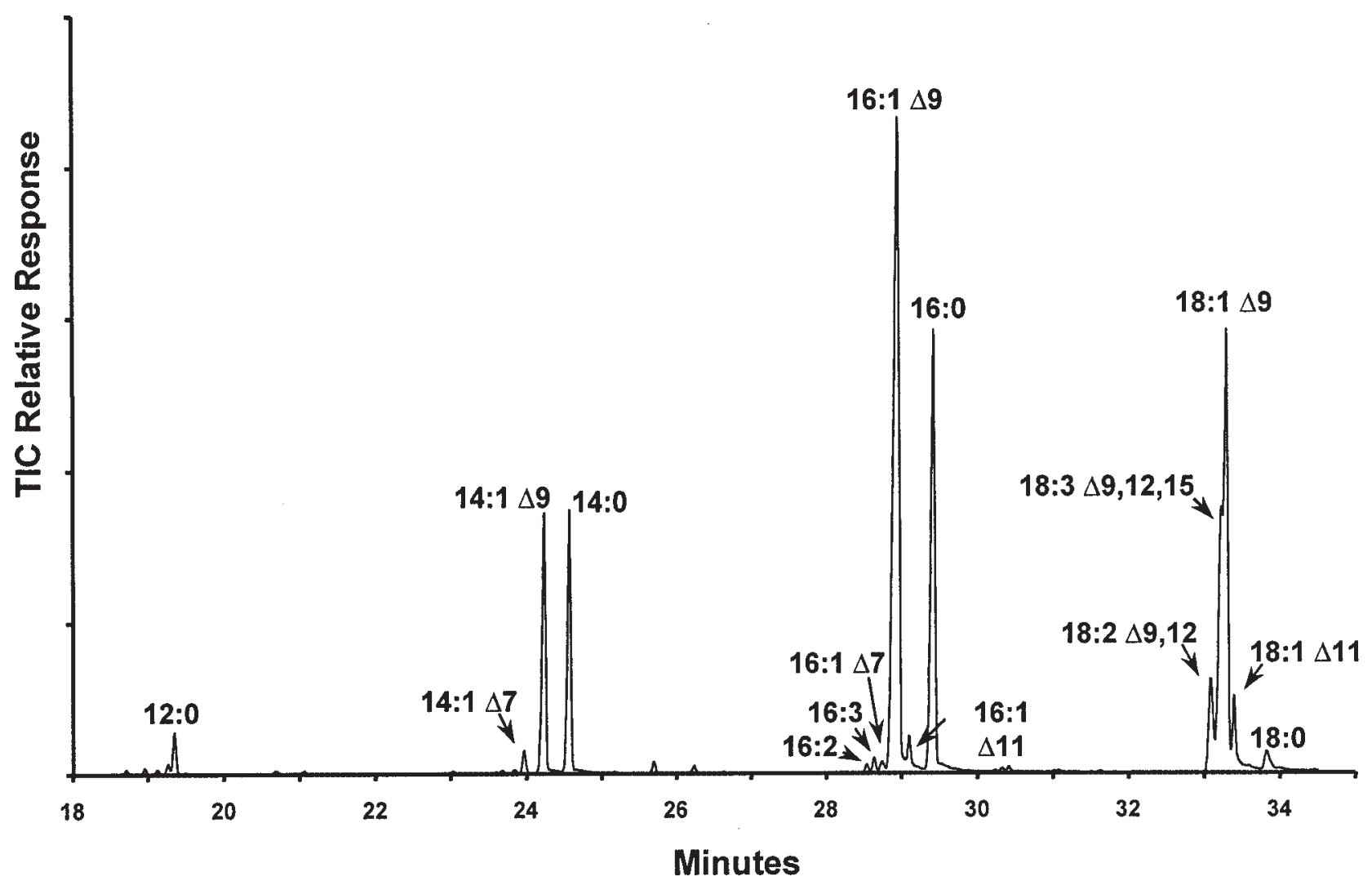

Fig. 2. A typical CGC-MS chromatogram of fatty acid on a J\&W DB-1 capillary column. Fatty acids are identitrimethylsilyl esters from a portion of the TAG fraction of fied according to Table 1. $10 \mathrm{M}$. rotundata prepupae (insect equivalent $=6.25 \times 10^{-5}$ )

TABLE 1. Identification and Percentage Composition of the Constituent Fatty Acids (as Their Trimethylsilyl Ester Derivatives) for the Triacylglycerols From M. rotundata prepupae

\begin{tabular}{|c|c|c|c|c|}
\hline \multirow[b]{2}{*}{ Peak Number ${ }^{\mathrm{a}}$} & \multicolumn{3}{|c|}{ Fatty acid component } & \multirow[b]{2}{*}{$\%$ Composition $^{\circ}$} \\
\hline & Short-hand notation ${ }^{b}$ & Common name of acid & Scientifc name & \\
\hline 1 & $12: 0$ & Lauric (La) & Dodecanoic acid & 1.2 \\
\hline 2 & $14: 1 \Delta 7$ & & Tetradec-7-enoic acid & 0.6 \\
\hline 3 & $14: 1 \Delta 9$ & Myristoleic (Mo) & Tetradec-9-enoic acid & 8.2 \\
\hline 4 & $14: 0$ & Myristic (M) & Tetradecanoic acid & 7.8 \\
\hline 5 & $16: 2$ & Palmitlinoleic $(\mathrm{PL})$ & Hexadecadienoic acid & 0.3 \\
\hline 6 & $16: 3$ & Palmitlinolenic (PLn) & Hexadecatrienoic acid & 1.2 \\
\hline 7 & $16: 1 \Delta 7$ & & Hexadec-7-enoic acid & 0.3 \\
\hline 8 & $16: 1 \Delta 9$ & Palmitoleic (Po) & Hexadec-9-enoic acid & 29.5 \\
\hline 9 & $16: 1 \Delta 11$ & & Hexadec-11-enoic acid & 1.5 \\
\hline 10 & $16: 0$ & Palmitic (P) & Hexadecanoic acid & 13.9 \\
\hline 11 & $18: 2 \Delta 9,12$ & Linoleic (L) & Octadec-9,12,15-dienoic acid & 5.0 \\
\hline 12 & $18: 3 \Delta 9,12,15$ & Linolenic (Ln) & Octadec-9,12,15-trienoic acid & 14.8 \\
\hline 13 & $18: 1 \Delta 9$ & Oleic $(0)$ & Octadec-9-enoic acid & 12.9 \\
\hline 14 & $18: 1 \Delta 11$ & Vaccenic & Octadec-11-enoic acid & 2.3 \\
\hline 15 & $18: 0$ & Stearic (S) & Octadecanoic acid & 0.6 \\
\hline
\end{tabular}

aNumbered peaks from the 19-34-min region of a GC-MS chromatogram (see Fig. 2) of individual fatty acid trimethylsilyl esters of TAG fatty acids.

${ }^{\mathrm{D} T}$ The numbers to the left and the right of the colon are the number of carbons and the number of double bonds, respectively, and the $\Delta$ numbers indicate the carbon positions for the double bond(s), counting from the carboxyl end.

'Percentage composition values were calculated from the quantities of fatty acid TMS derivatives as determined from the GC-MS integrated peak area for each component and standard curves for authentic standards as described in Materials and Methods. 
of the acid moiety, the double bond occurs between carbons $\mathrm{n}$ and $\mathrm{n}+1$ in the molecule (Andersson and Holman, 1974). For GC-MS analysis of the M. rotundata TAG fatty acids, the mass spectrum of the major component (16:1) was consistent for the pyrrolidine derivative of hexadec-9-enoic acid (palmitoleic acid) with a molecular ion at $\mathrm{m} / z$ 307, a base peak at $m / z 113$ for the McLafferty rearrangement of all fatty acid pyrrolidides, and diagnostic fragment ions 12 amu apart at $m / z 196$ and $m / z$ 208 for a double bond at the $\Delta^{9}$ position. The other major monounsaturated components, $14: 1$ and $18: 1$, with mass spectra showing molecular ions at $m / z 279$ and $m / z 335$, respectively, had the same diagnostic fragments ions 12 amu apart for a $\Delta^{9}$ double bond position. For the GC-MS peak of 18:2, a molecular ion at $m / z 333$ and characteristic series of fragment ions at $m / z 196,208$ and $m / z$ 250, 262 for double bond positions at $\Delta^{9}$ and $\Delta^{12}$, respectively, are consistent for the pyrrolidine derivative of octadec-9,12-dienoic acid (linoleic acid). A molecular ion at $m / z 331$ and an overall mass spectral fragmentation pattern that was identical to the mass spectrum for the pyrrolidide of authentic octadec-9,12,15-trienoic acid strongly suggested that the 18:3 peak from $M$. rotundata TAG fatty acids was the $\Delta^{9,12,15}$ fatty acid, linolenic acid. For the three monounsaturated fatty acids, $14: 1,16: 1$, and $18: 1$, small quantities of other double bond positional components were identified: $\Delta^{7}$ positions for 14:1 and 16:1, and $\Delta^{11}$ positions for $16: 1$ and $18: 1$ (see Table 1 for \% compositions). Molecular ions at $m / z 305$ and 303 confirmed the presence of 16:2 and 16:3 for the small peaks that eluted before the 16:1 $\Delta^{7}$ peak (Fig. 2), but double bond positions could not be clearly established from the mass spectral fragmentation patterns of their pyrrolidine derivatives.

\section{HPLC-MS Identification of Triacylglycerols}

Intact TAG were separated using an HPLC column packed with silica material derivatized with a cation exchange resin in the $\mathrm{Ag}^{+}$ionic form. The mixture of TAG components from $M$. rotundata prepupae TAG fraction were eluted from the col- umn as groups (nine) of peaks according to degree of unsaturation, and within each group according to fatty acid chain length (Fig. 3). TAG components were structurally identified by interpretation of their electron ionization mass spectra. Diagnostic ions included the molecular ion, the diacylglyceride (DAG) ions (with the most intense ion corresponding to the loss of the most unsaturated fatty acid), and the monoglyceride (MAG) ions and acyl ions for each fatty acid constituent. Many of the peaks in the TAG fraction, as shown in Figure 3, contained one or more TAG components and a single mass spectrum contained diagnostic ions for more than one TAG component. An improvement in TAG component resolution was achieved by configuring two of the HPLC columns in series (data not shown) and the acquisition of multiple mass spectral scans across each HPLC peak was used to identify individual TAG components and their fatty acid constituents.

The first group of eluting peaks (peaks 1 and 2 ) in Figure 3 was characterized as TAGs with two saturated and one monounsaturated fatty acid constituents associated with each TAG. Three TAG components were identified in both peaks (Table 2) and, from the relative intensities of diagnostic MAG and DAG ions, the main TAG components for peaks 1 and 2 were identified as myristoyl palmitoyl oleoyl glycerol (MPO) and myristoyl palmitoyl palmitoleoyl glycerol (MPPo), respectively (Table 2) (mass spectral data not shown). In terms of order of elution for those TAG with the same degree of unsaturation, the TAG with fatty acid constituents that possess the longer carbon chain lengths elute first. Thus, MPO eluted before MPPo. The major TAG peaks in HPLC-MS chromatograms of the TAG fraction were peaks 7 and 8 within Group 2 and peak 27 of Group 4 (Fig. 3) that consisted of one saturated and two monounsaturated constituents (Group 2), and one saturated, one monounsaturated, and one triunsaturated constituent (Group 4), respectively. The largest peak of Group 2 (peak 7) was identified as mainly palmitoyl palmitoleoyl palmitoleoyl glycerol (PPoPo). The other major Group 2 peak (peak 8) was identified as myristoyl palmitoleoyl palmitoleoyl glycerol 


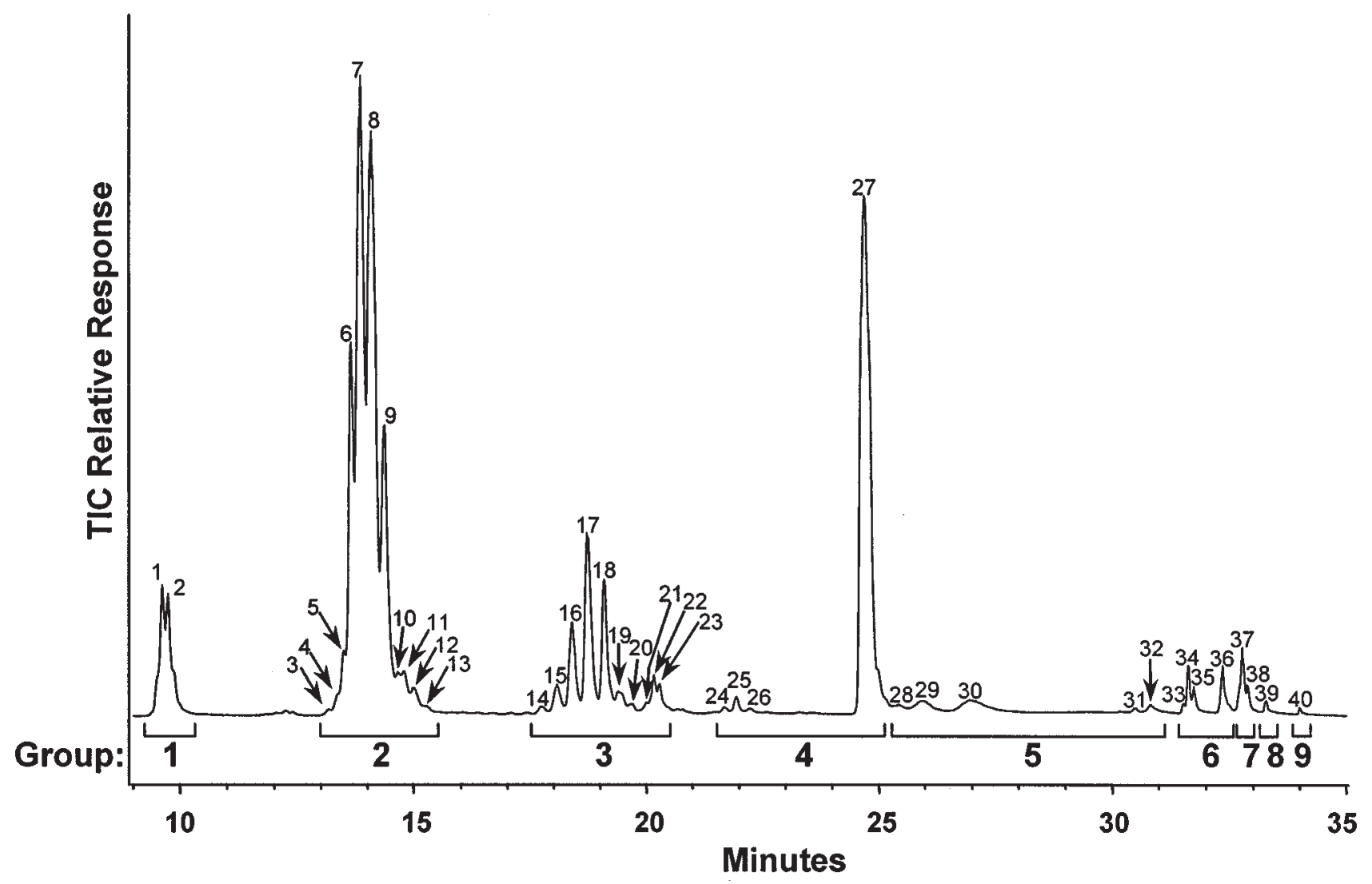

Fig. 3. A typical HPLC-MS chromatogram of a portion of the intact triacylglycerol fraction from five $M$. rotundata prepupae (insect equivalent $=0.067$ ). TAG components mixtures were eluted from the column as groups of peaks according to degree of unsaturation, and within each group according to fatty acid chain length. Group num-

(MPoPo). Peaks 6 and 9 in Group 2 were identified as palmitoyl palmitoleoyl oleoyl (PPoO) and myristoleoyl palmitoyl palmitoleoyl glycerol (MoPPo), respectively. The major peak 27 (Group 4) consisted of multiple TAG components, palmitoyl oleoyl linolenoyl glycerol (POLn), palmitoyl palmitoleoyl linolenoyl glycerol (PPoLn), and myristoyl palmitoleoyl linolenoyl glycerol (MPoLn). The three major peaks in Group 3 (peaks 16-18) consisted of three monounsaturated constituents and were identified as PoPoO, PoPoPo, and MoPoPo, respectively. Linoleic acid (L), as a diunsaturated fatty acid, was a common fatty acid constituent of TAG identified in peaks 21-26. The triunsaturated fatty acid linolenic acid (Ln) was either a single constituent or bers represent the total number of double bonds for the three acyl constituents of each TAG component (i.e., Group 2 consists of peaks of TAG components with a total of two double bonds: one saturated fatty acid and two monounsaturated fatty acid constituents).

multiple constituents of the TAG peaks 27-40, except for the TAG components of peaks 28 and 31 that had a palmitlinolenoyl (PLn) moiety (Table 2 ). Interpretation of mass spectra indicated the presence of positional isomers for the moieties of peaks 19 and 20 (MoPoMo and MoMoPo, respectively) and for two TAG components of peak 37 (LnPoLn and PoLnLn) (Table 2).

\section{DISCUSSION}

Megachile rotundata prepupae possessed copious quantities of stored lipid: $20 \%$ of the wet weight. In contrast, most insect species contain less than $10 \%$ of wet weight as lipid (Fast, 1966, 1970) and 
TABLE 2. Identification of M. rotundata Triacylglycerols and Their Fatty Acid Constituents

\begin{tabular}{|c|c|c|c|c|c|}
\hline $\begin{array}{l}\text { Peak } \\
\text { number }^{\mathrm{a}} \\
\end{array}$ & $\begin{array}{c}\text { Triacylglycerols } \\
\text { and fatty acid } \\
\text { constituents }^{b}\end{array}$ & Scientific name & $\begin{array}{l}\text { Peak } \\
\text { number }^{\mathrm{a}}\end{array}$ & $\begin{array}{l}\text { Triacylglycerols } \\
\text { and fatty acid } \\
\text { constituents }^{b}\end{array}$ & Scientific name \\
\hline Group 1 & 1 Double bond & & 25 & PoPoL & Palmitoleoyl palmitoleoyl linoleoyl glycerol \\
\hline \multirow[t]{3}{*}{1} & MPO & Myristoyl palmitoyl oleoyl glycerol & 26 & MoPoL & Myristoleoyl palmitoleoyl linoleoyl glycerol \\
\hline & MMO & Myristoyl myristoyl oleoyl glycerol & 27 & POLn & Palmitoyl oleoyl linolenoyl glycerol \\
\hline & LaPO & Lauroyl palmitoyl oleoyl glycerol & & PPoLn & Palmitoyl palmitoleoyl linolenoyl glycerol \\
\hline \multirow[t]{3}{*}{2} & MPPo & Myristoyl palmitoyl palmitoleoyl glycerol & & MPoLn & Myristoyl palmitoleoyl linolenoyl glycerol \\
\hline & MMPo & Myristoyl myristoyl palmitoleoyl glycerol & & PPoPLn & Palmitoyl palmitoleoyl palmitlinolenoyl \\
\hline & LaM0 & Lauroyl myristoyl oleoyl glycerol & & & glycerol \\
\hline Group 2 & 2 Double bonds & & Group 5 & 5 Double bonds & \\
\hline 3 & P00 & Palmitoyl oleoyl oleoyl glycerol & 28 & OOLn & Oleoyl oleoyl linolenoyl glycerol \\
\hline 5 & M00 & Myristoyl oleoyl oleoyl glycerol & 29 & PoOLn & Palmitoleoyl oleoyl linolenoyl glycerol \\
\hline 6 & PPo0 & Palmitoyl palmitoleoyl palmitoleoyl glycerol & 30 & PoPoLn & Palmitoleoyl palmitoleoyl linolenoyl glycero \\
\hline 7 & PPoPo & Palmitoyl palmitoleoyl palmitoleoyl glycerol & & MoPoLn & Myristoleoyl palmitoleoyl linolenoyl glycero \\
\hline 8 & MPoPo & Myristoyl palmitoleoyl palmitoleoyl glycerol & 31 & PoPoPLn & Palmitoleoyl palmitoleoyl palmitlinolenoyl \\
\hline 9 & MoPPo & Myristoleoyl palmitoleoyl palmitoleoyl glycerol & & & glycerol \\
\hline 10 & MMoPo & Myristoyl myristoleoyl palmitoleoyl glycerol & 32 & PLLn & Palmitoyl linoleoyl linolenoyl glycerol \\
\hline 11 & MoMoP & Myristoleoyl myristoleoyl palmitoyl glycerol & Group 6 & 6 Double bonds & \\
\hline Group 3 & 3 Double bonds & & 33 & OLLn & Oleoyl linoleoyl linolenoyl glycerol \\
\hline 14 & 000 & Oleoyl oleoyl oleoyl glycerol & 34 & PoLLn & Palmitoleoyl linoleoyl linolenoyl glycerol \\
\hline 15 & Po00 & Palmitoleoyl oleoyl oleoyl glycerol & 35 & MoLLn & Myristoleoyl linoleoyl linolenoyl glycerol \\
\hline 16 & PoPoO & Palmitoleoyl palmitoleoyl oleoyl glycerol & 36 & PLnLn & Palmitoyl linolenoyl linolenoyl glycerol \\
\hline 17 & PoPoPo & Palmitoleoyl palmitoleoyl palmitoleoyl glycerol & & MLnLn & Myristoyl linolenoyl linolenoyl glycerol \\
\hline 18 & MoPoPo & Myristoleoyl palmitoleoyl palmitoleoyl glycerol & Group 7 & 7 Double bonds & \\
\hline 19 & MoPoMo\} Isomer ${ }^{c}$ & Myristoleoyl palmitoleoyl myristoleoyl glycerol & 37 & OLnLn & Oleoyl linolenoyl linolenoyl glycerol \\
\hline 20 & MoMoPo\} Isomer ${ }^{c}$ & Myristoleoyl myristoleoyl palmitoleoyl glycerol & & LnPoLn\} Isomer ${ }^{c}$ & Linolenoyl palmitoleoyl linolenoyl glycerol \\
\hline 21 & POL & Palmitoyl oleoyl linoleoyl glycerol & & PoLnLn\} Isomer ${ }^{c}$ & Palmitoleoyl linolenoyl linolenoyl glycerol \\
\hline 22 & PPoL & Palmitoyl palmitoleoyl linoleoyl glycerol & 38 & MoLnLn & Myristoleoyl linolenoyl linolenoyl glycerol \\
\hline 23 & MPoL & Myristoyl palmitoleoyl linoleoyl glycerol & Group 8 & 8 Double bonds & \\
\hline & LaPoL & Lauroyl palmitoleoyl linoleoyl glycerol & 39 & LLnLn & Linoleoyl linolenoyl linolenoyl glycerol \\
\hline Group 4 & 4 Double bonds & & Group 9 & 9 Double bonds & \\
\hline 24 & PoOL & Palmitoleoyl oleoyl linoleoyl glycerol & 40 & LnLnLn & Linolenoyl linolenoyl linolenoyl glycerol \\
\hline
\end{tabular}

apeaks, numbered according to their order of elution from HPLC-MS analysis of triacylglycerol fraction as indicated in Figure 3.

${ }^{b}$ For mixed TAGs, the position of fatty acid constituents on carbons 1, 2, and 3 of glycerol was not established. A consistent system was used for positioning and naming: shorter carbon chain lengths first, then by degree of unsaturation (e.g., MPPo).

'The position of fatty acid constituents for positional isomer triacylglycerols was based on interpretation and abundance of diagnostic monoglyceride and diacylglyceride mass spectral fragment ions.

for the honeybee (Apis mellifera L.), lipids as percentage of fresh weight range between 5.9 and $8.6 \%$ for both larval and pupal drones, workers, and queens (Robinson and Nation, 1970). Analyses of total lipid extracts from a variety of insect species have shown that the predominant lipid class is TAG (Downer, 1985) and that fat body tissue is the primary site of TAG storage (Downer, 1985; Keeley, 1985). In this study, chromatographic analyses revealed that $88 \%$ of the extractable internal lipids from $M$. rotundata were associated with the TAG fraction and ultrastructural studies revealed lipid storage within very large vacuoles (Fig. 1) located within apparent fat body cells. For the TAG fraction, the fatty acid composition and double bond location was established by CGC-MS of the fatty acids as their TMS and pyrrolidide derivatives, respectively. We chose to analyze the fatty acids as their TMS derivatives because no clean up was required after derivatization and, losses of the more volatile polyunsaturates were minimal. A total of 15 saturated and unsaturated fatty acid structures were identified (Fig. 2 and Table 1).

The majority of the observed TAG fatty acids were unsaturated $(76 \%)$ and the major fatty acid constituent (31.4\%) was the monounsaturated fatty acid, palmitoleic acid (16:1, hexadec-9-enoic acid). A high ratio of unsaturated/saturated fatty acids in storage lipids has been associated to insect wintering or to species adapted to cold climates, and has 
been interpreted as a mechanism to maintain the appropriate fluidity of these lipid reserves (Ohtsu et al., 1993; Joanisse and Storey, 1996; Kostal and Simek, 1998). The function of increased fatty acid unsaturation, however, remains unclear, as high unsaturated/saturated fatty acid ratios are found in species that use glycogen, rather than TAGs, as their main energy reserve during winter or summer diapause (Joanisse and Storey, 1996; Bennett et al., 1997; Kostal et al., 1998), as well as in species in which TAGs are the main winter energy source (Pullin, 1987; Kurthiala and Chippendale, 1989; Ohtsu et al., 1993, 1995; Joanisse and Storey, 1996). Weight loss in M. rotundata is almost negligible throughout late summer and winter, and only increases once prepupae are exposed to warm temperatures in the spring (Kemp et al., 2004). Again, this pattern is found both in species using glycogen and in species using TAGs as their main winter energy source (Joanisse and Storey, 1996).

In terms of fatty acid distribution in insects, 16:1 as the major fatty acid is not a common occurrence. Insects contain a $\Delta^{9}$ desaturase that desaturates 16:0 and 18:0 fatty acids to $\Delta^{9}$ monounsaturates (Stanley-Samuelson et al., 1988); however, for most insects the quantities of 16:1 are minor compared to 18:1 (Downer, 1985; StanleySamuelson et al., 1988). High proportions of 16:1 fatty acids have also been found in some Heteroptera (Spike et al., 1991; Bashan et al., 2002). For the total lipids from Homoptera, amounts of 16:1 were minor in aphids, ranging between $0.6-2.6 \%$ (Dillwith et al., 1993) and, for the internal lipids of nymph and adult whiteflies, 16:1 amounts were only $1-2 \%$ (Buckner and Hagen, 2003). For the Hymenoptera, the major fatty acid was 18:1 (46\%) and the amount of 16:1 was only 4\% (Downer, 1985).

For the TAG fatty acids from $M$. rotundata prepupae, we also detected small quantities of 16:2 and 16:3 (Fig. 2 and Table 1), and an intact TAG component was identified with a 16:3 constituent (Peak 31; Fig. 3 and Table 2). To our knowledge, this is the first reported occurrence of $16: 2$ and 16:3 fatty acids in insects, although a conjugated 16:2 (10,12-hexadecadienoic acid) was observed as a precursor of pheromone biosynthesis in female silkworm moths (Ando et al., 1996). For other organisms, quantities of 16:2 have been observed in yeast cells (Ito et al., 1983; Brown et al., 1998) and marine algae (Viso and Marty, 1993), and 16:3 was present in plant leaves (Horiguchi et al., 1996) and algae (Viso and Marty, 1993).

The predominance of 16:1 (palmitoleic acid (Po)) for the TAG fatty acids was also reflected by structural identifications of intact TAGs as determined by HPLC-MS. Identification of fatty acid constituents for each TAG was made from interpretable mass spectra (Table 2), but the fatty acid position on carbon 1, 2, or 3 of the glycerol molecule was not determined. The TAG fraction from $M$. rotundata prepupae was shown to be a complex mixture of more than 40 TAG components (Fig. 3 and Table 2) with different combinations of the 15 fatty acid constituents, as shown in Figure 2 and Table 1 . The major fatty acid constituent, palmitoleic acid (Po), was identified in about $75 \%$ of the identified TAG components (Table 2). As indicated by total ion current peak area in the typical HPLC-MS chromatogram of prepupae TAG (Fig. 3), the major peaks were nos. 6-9 and 27, and all identified TAG components had one or two palmitoleic acid constituents, except for the $\mathrm{POL}_{\mathrm{N}}$ component within peak 27 (see Table 2).

Although glycerol is the most commonly encountered low-molecular weight cryoprotectant in insects (Lee and Denlinger, 1991), and has been found in M. rotundata, the contributions of stored lipids (TAGs) to cold hardiness in wintering insects like $M$. rotundata has not been clearly established. There is, however, an association between the synthesis and sequestration of large amounts of stored lipid in cold-hardy insects and a decrease in water content. Many insects reduce their water content before winter, at a time when cryoprotectants (glycerol, sorbitol, etc.) typically increase (Ring, 1981; Cannon and Block, 1988; Lee and Denlinger, 1991), although this reduction may only reflect increases in lipid stores (Danks, 2000). For example, in studies of the boll weevil, Anthonomus grandis Boh., an increase in fat correlated to a decrease in body water (Brazzel and Newsom, 1959). 
For A. grandis from different latitudes, Keeley et al. (1977) suggested that the lower lipid content and associated higher water content in diapausing weevils from warmer climates may be necessary to survive the arid climate of southern latitudes. Larvae of the gall fly larva, Eurosta solidaginis, have been extensively used as a model system for studying freeze tolerance and biochemical adaptations (Storey et al., 1981; Storey and Storey, 1986, 1988). Intermediary metabolism, correlated with low temperature acclimation and winter survival studies with E. solidaginis, revealed that the large total glyceride reserves (an average of $312 \mu \mathrm{mol} / \mathrm{g}$ wet weight) and larval weight were not altered over the course of the winter season (Storey and Storey, 1986). No correlations between lipid levels and the synthesis or degradation of polyols were observed, suggesting that E. solidaginis lipid reserves were not involved with cryoprotectant production.

$M$. rotundata prepupae overwintering in temperate regions frequently spend more than 9 months in a non-feeding state. This includes three more or less distinct periods, diapause, cold-mediated postdiapause, and springtime pupation and adult emergence (Kemp et al., 2004), during which these insects are faced with the multiple tasks of maintaining basal metabolic processes, surviving extreme winter conditions (temperatures as low as $-30^{\circ} \mathrm{C}$ ) and, perhaps equally difficult, frequent diurnal freeze thaw cycles. Although insects possess diverse patterns of energy/cryoprotectant intermediary metabolites (Lee, 1991; Table 2.1), our results for $M$. rotundata appear consistent with the argument (Downer, 1985) that TAGs, whose major repository is the fat body in insects, are frequently associated with species that undergo lengthy periods of metabolic activity (for example, diapause or migratory flights) or that have nonfeeding stages of development (e.g., pupa, adult). From a bioenergetic standpoint, TAGs appear to offer several advantages over glycogen, another frequently encountered metabolic reserve (Lee, 1991; Table 2.1), including a higher caloric content per unit weight, the liberation of two times more metabolic water than is obtained from carbohydrates upon oxidation, and the capacity for storage in an- hydrous form, instead of the more bulky hydrated form as is the case for glycogen (Downer, 1985).

The water content in $M$. rotundata prepupae at the end of the wintering period was $39.1 \pm 3.5 \%$ ( $n=9)$ and, even though the water content has not been established for prepupae entering diapause, recent findings have indicated very low $\mathrm{O}_{2}$ consumption and static weight for overwintering M. rotundata prepupae, followed by increased $\mathrm{O}_{2}$ consumption and more rapid weight losses, during incubation, as individuals pupate and ultimately emerge as adults (Kemp et al., 2004). Thus, the large quantities of intracellular-stored lipid that we observed likely have a dual role in the overwintering physiology of $M$. rotundata. In addition to reducing intracellular water and thus increasing concentrations of cryoprotectants, lipid stores in M. rotundata likely have a very important bioenergetic function in support of basal metabolic functioning during diapause and subsequent development through the pupa and emerged adult. Future studies will focus on correlating lipid and water levels with metabolic activities, including the accumulation of other cryoprotectants, during prewintering, wintering, and post-wintering stages of M. rotundata development.

\section{ACKNOWLEDGMENTS}

In Fargo, ND, we thank Carlee O'Dell and Rita Ruud for technical assistance and a special thank you to Marcia Hagen for chromatographic analyses, mass spectral interpretations, and data presentations. In Logan, UT, we appreciate the technical assistance of Joyce Knoblett. At the Electron Microscope Center at North Dakota State University, Fargo, ND, we thank Dr. Thomas Freeman and Scott Payne for sample preparations and microscopic analyses. Critical reviews of the manuscript by Drs. Brady Vick, Stephen Foster, Heldur Hakk, and Thomas Freeman are appreciated.

\section{LITERATURE CITED}

Andersson BA, Holman RT. 1974. Pyrrolidides for mass spectrometric determination of the position of the double bond in monounsaturated fatty acids. Lipids 9:185-190. 
Ando T, Kasuga K, Yajima Y, Kataoka H, Suzuki A. 1996. Termination of sex pheromone production in mated females of the silkworm moth. Arch Insect Biochem Physiol 31:207-218.

Bashan M, Akbas H, Yurdacok K. 2002. Phospholipid and triacylglycerol fatty acid composition of major life stages of sunn pest, Eurygaster integriceps (Heteroptera: Scutellaridae). Comp Biochem Physiol 132B:375-380.

Bennett VA, Pruitt NL, Lee RE. 1997. Seasonal changes in fatty acid composition associated with cold-hardening in third instar larvae of Eurosta solidaginis. J Comp Physiol B 167:249-255.

Bosch J, Kemp WP. 2002. Developing and establishing bee species as crop pollinators: the example of Osmia spp. Bul Entomol Res 92:3-16.

Bosch J, Maeta Y, Rust RW. 2001. A phylogenetic analysis of nesting behavior in the genus Osmia (Hymenoptera: Megachilidae). Ann Entomol Soc Am 94:617-627.

Brown AP, Dann R, Bowra S, Hills MJ. 1998. Characterization of expression of a plant oleate desaturase in yeast. J Am Oil Chem Soc 75:77-82.

Brazzel JR, Newsom LD. 1959. Diapause in Anthonomus grandis. Boh J Econ Ent 52:603-611.

Buckner JS, Hagen MM. 2003. Triacylglycerol and phospholipid fatty acids of the silverleaf whitefly: composition and biosynthesis. Arch Insect Biochem Physiol 53:66-79.

Buckner JS, Hagen MM, Nelson DR. 1999. The composition of the cuticular lipids from nymphs and exuviae of the silverleaf whitefly, Bemisia argentifolii. Comp Biochem Physiol 124B:201-207.

Buckner JS, Freeman TP, Ruud RL, Chu CC, Henneberry TJ. 2002. Characterization and functions of the whitefly egg pedicel. Arch Insect Biochem Physiol 49:22-33.

Cannon RJC, Block W. 1988. Cold tolerance of microarthropods. Biol Rev 63:23-77.

Danks HV. 1987. Insect dormancy: an ecological perspective. Biological Survey of Canada Monograph Series No 1, Ottawa.

Danks HV. 2000. Dehydration in dormant insects. J Insect Physiol 46:837-852.

Dillwith JW, Neese PA, Brigham DL. 1993. Lipid biochemistry in aphids. In: Stanley-Samuelson DW, Nelson DR, editors.
Insect lipids: chemistry, biochemistry and biology. Lincoln, Nebraska: University of Nebraska Press. p 389-434.

Downer RGH. 1985. Lipid metabolism. In: Kerkut GA, Gilbert LI, editors. Comprehensive insect physiology, biochemistry and pharmacology. Oxford: Pergamon Press. p 77-113.

Fast PG. 1966. A comparative study of the phospholipids and fatty acids of some insects. Lipids 1:209-215.

Fast PG. 1970. Insect lipids. In: Holman RT, editor. Progress in the chemistry of fats and other lipids, vol. 11. Oxford: Pergamon Press. p 181-242.

Folch J, Lee M, Slone-Stanley GH. 1957. A simple method for the isolation and purification of total lipids from animal tissues. J Biol Chem 226:497-509.

Gauld ID, Bolton B. 1988. The Hymenoptera. Oxford: Oxford University Press, 332 p.

Gusta LV. 1982. Supercooling in Megachile and Pteromalus venustus larvae in relation to temperature. In: Rank GH, editor. Proceedings of the First International Symposium on Alfalfa Leafcutting Bee Management, 16-18 August 1982, University of Saskatchewan, Saskatoon. p 20-34.

Hamilton JG, Comai K. 1988. Rapid separation of neutral lipids, free fatty acids and polar lipids using prepacked silica Sep-Pak columns. Lipids 23:1146-1149.

Horiguchi G, Kodama H, Nishimura M, Iba K. 1996. Role of omega-3 fatty acid desaturases in the regulation of the level of trienoic fatty acids during leaf cell maturation. Planta 199:439-442.

Ito H, Fukuda Y, Murata K, Kimura A. 1983. Transformation of intact yeast cells treated with alkali cations. J Bacteriol 153:163-168.

Joanisse DR, Storey KB. 1996. Fatty acid content and enzymes of fatty acid metabolism in overwintering cold-hardy gall insects. Physiol Zool 69:1079-1095.

Johansen CA, Eves JD. 1973. Effect of chilling, humidity and seasonal conditions on emergence of the alfalfa leafcutting bee. Environ Entomol 2:23-26.

Keeley LL. 1985. Physiology and biochemistry of the fat body. In: Kerkut GA, Gilbert LI editors. Comprehensive insect physiology, biochemistry and pharmacology. Oxford: Pergamon Press. p 211-248. 
Keeley LL, Moody DS, Lynn D, Joiner RL, Vinson SB. 1977. Succinate-cytochrome $c$ reductase activity and lipids in diapause and non-diapause Anthonomus grandis from different latitudes. J Insect Physiol 23:231-234.

Kemp WP, Bosch J. 2000. Development and emergence of the alfalfa pollinator Megachile rotundata (Hymenoptera: Megachilidae). Ann Entomol Soc Am 93: 904-911.

Kemp WP, Bosch J. 2001. Postcocooning temperatures and diapause in the alfalfa pollinator Megachile rotundata (Hymenoptera: Megachilidae). Ann Entomol Soc Am 94: 244-250.

Kemp WP, Bosch J, Dennis B. 2004. Life cycle oxygen consumption in the prepupa-wintering bee, Megachile rotundata (F.) and the adult-wintering bee Osmia lignaria Say (Hymenoptera: Megachildae). Ann Entomol Soc Am $97: 161-170$.

Kostal V, Simek P. 1998. Changes in fatty acid composition of phospholipids and triacylglycerols after cold-acclimation of an aestivating insect prepupa. J Comp Physiol B 168:453-460.

Kostal V, Sula J, Simek P. 1998. Physiology of drought tolerance and cold hardiness of the Mediterranean tiger moth Cymbalophora pudica during summer diapause. J Insect Physiol 44:165-173.

Krunic MD, Brajkovic MM, Richards KW. 1982. Some aspects of cold-hardiness in Megachile rotundata (Hymenoptera: Megachildae), In: Rank GH, editor. Proceedings of the First International Symposium on Alfalfa Leafcutting Bee Management, 16-18 August 1982, University of Saskatchewan, Saskatoon. p 35-40.

Kurthiala A, Chippendale GM. 1989. Relationship between the fatty acids of fat body triacylglycerol and lipophorin diacylglycerol of non-diapause and diapause larvae of the corn borer, Diatraea grandiosella. Arch Insect Biochem Physiol 12:123-131.

Lee RE. 1991. Principles of insect low temperature tolerance. In: Lee RE, Denlinger DL, editors. 1991. Insects at low temperature. New York: Chapman and Hall. p 17-46.

Lee RE, Denlinger DL, editors. 1991. Insects at low temperature. New York: Chapman and Hall. 513 p.

Murrell D. 1991. A calendar of incubation for alfalfa leafcutting bees. Sask Agric Newslett 13.
Ohtsu T, Katagiri C, Kimura MT, Hori SH. 1993. Cold adaptations in Drosophila: qualitative changes of triacylglycerols with relation to overwintering. J Biol Chem 268:18301834.

Ohtsu T, Kimura MT, Hori SH. 1995. The influence of eclosion timing on winter survival and triacylglycerol accumulation in four temperature species of Drosophila. Physiol Entomol 20:248-252.

Parker FD, Tepedino VJ. 1982. Maternal influence on diapause in the alfalfa leafcutting bee (Hymenoptera: Megachilidae). Ann Entomol Soc Am 75:407-410.

Peterson SS, Baird CR, Bitner RM. 1992. Current status of the alfalfa leafcutting bee, Megachile rotundata, as a pollinator of alfalfa seed. Bee Sci 2:135-142.

Pullin AS. 1987. Adult feeding time, lipid accumulation, and overwintering in Aglais urticae and Inachis io (Lepidoptera: Nymphalidae). J Zool Lond 211:631-641.

Rank GH, Goerzen DW. 1982. Shelter design and hive placement for optimizing alfalfa leafcutter bee activity. In: Rank $\mathrm{GH}$, editor. Proceedings of the First International Symposium on Alfalfa Leafcutting Bee Management, 16-18 August 1982, University of Saskatchewan, Saskatoon. p. $144-151$.

Rank GH, Rank FP. 1989. Diapause intensity in a French univoltine and a Saskatchewan commercial strain of Megachile rotundata (Fab.). Can Entomol 121:141-148.

Richards KW. 1984. Alfalfa leafcutter bee management in Western Canada. Agriculture Canada Publication 1495/E. Agriculture Canada, Ottawa.

Richards KW, Whitfield GH. 1988. Emergence and survival of leafcutter bees, Megachile rotundata, held at constant incubation temperatures (Hymenoptera: Megachilidae). J Apic Res 27:197-204.

Richards KW, Whitfield GH, Schaalje GB. 1987. Effects of temperature and duration of winter storage on survival and period of emergence for the alfalfa leafcutter bee (Hymenoptera: Megachilidae). J Kansas Entomol Soc 60:70-76.

Ring RA. 1981. The physiology and biochemistry of cold tolerance in arctic insects. J Thermal Biol 6:219-229.

Robinson FA, Nation JL. 1970. Long-chain fatty acids in honeybees in relation to sex, caste, and food during development. J Apicult Res 9:121-127. 
Spike BP, Wright RJ, Danielson SD, Stanley-Samuelson DW. 1991. The fatty acid composition of phospholipids and triacylglycerols from two chinch bug species Blissus leucopterus leucopterus and B. iowensis (Insecta: Hemiptera; Lygaeidae) are similar to the characteristic dipteran pattern. Comp Biochem Physiol 99B:799-802.

Stanley-Samuelson DW, Jurenka RA, Cripps C, Blomquist GJ, deRenobales M. 1988. Fatty acids in insects: composition, metabolism and biological significance. Arch Insect Biochem Physiol 9:1-33.

Stephen WP. 1981. The design and function of field domiciles and incubators for leafcutting bee management (Megachile rotundata (Fabricius)). Bulletin 654. Oregon State University. Agricultural Experiment Station, Corvallis, OR.
Storey JM, Storey KB. 1986. Winter survival of the gall fly larva, Eurosta solidaginis: profiles of fuel reserves and cryoprotectants in a natural population. J Insect Physiol 32:549-556.

Storey KB, Storey JM. 1988. Freeze tolerance in animals. Physiol Rev 68:27-84.

Storey KB, Baust JG, Storey JM. 1981. Intermediary metabolism during low temperature acclimation in the overwintering gall fly larva, Eurosta solidaginis. J Comp Physiol 144:183-190.

Taséi JN, Masure MM. 1978. Sur quelques facteurs influençant le dévélopment de Megachile pacifica Panz. (Hymenoptera: Megachilidae). Apidologie 9:273-290.

Viso AC, Marty JC. 1993. Fatty acids from 28 marine microalgae. Phytochemistry 34:1521-1533. 